molecular bases of the TNF signaling. Given its important contribution to TNF-induced JNK activation, AIP1 may represent a suitable target for possible therapeutic applications in human diseases characterized by increased TNF- $\alpha$-mediated apoptosis.

\section{Acknowledgments}

This work was supported by grants from the NIH (DK 63947) and the Mayo Foundation.

1. Wallach, D., et al. 1999. Tumor necrosis factor receptor and Fas signaling mechanisms. Ann. Rev. Immunol. 17:331-367.

2. McDermott, M.F. 2001. TNF and TNFR biology in health and disease. Cell. Mol. Biol. 47:619-635.

3. Wang, C.Y., Mayo, M.W., Korneluk, R.G., Goeddel, D.V., and Baldwin, A.S. 1998. NF-кB antiapoptosis: induction of TRAF 1 and TRAF 2 and c-IAP1 and c-IAP2 to suppress caspase- 8 activation. Science. 281:1680-1683.

4. Kischkel, F.C., et al. 2001. Death receptor recruitment of endogenous caspase- 10 and apoptosis initiation in the absence of caspase-8. J. Biol. Chem. 276:46639-46646.

5. Wajant, H., and Scheurich, P. 2001. Tumor necrosis factor receptor-associated factor (TRAF) 2 and its role in TNF signaling. Int. J. Biochem. Cell. Biol. 33:19-32.

6. Ip, Y.T., and Davis, R.J. 1998. Signal transduction by the c-jun $\mathrm{N}$-terminal kinase (JNK): from inflammation to development. Curr. Opin. Cell Biol. 10:205-219.

7. Ono, K., and Han, J. 2000. The p38 signal transduction pathway: activation and function. Cell Signal. 12:1-13.

8. Nishitoh, H., et al. 1998. ASK1 is essential for JNK/SAPK activation by TRAF2. Mol. Cell. 2:389-395.

9. Chang, H.Y., Nishitoh, H., Yang, X., Ichijo, H., and Baltimore, D. 1998. Activation of apoptosis signal-regulating kinase 1 (ASK1) by the adapter protein Daxx. Science. 281:1860-1863.

10. Ichijo, H., et al. 1997. Induction of apoptosis by ASK1, a mammalian MAPKKK that activates SAPK/JNK and p38 signaling pathways. Science. 275:90-94.

11. Liu, H., Nishitoh, H., Ichijo, H., and Kyriakis, J.M. 2000. Activation of apoptosis signal-regulating kinase 1 (ASK1) by tumor necrosis factor receptor-associated factor 2 requires prior dissociation of the ASK1 inhibitor thioredoxin. Mol. Cell. Biol. 20:2198-2208

12. Saitoh, M., et al. 1998. Mammalian thioredoxin is a direct inhibitor of apoptosis signal- regulat ing kinase (ASK) 1. EMBOJ. 17:2596-2606.

13. Zhang, L., Chen, J., and Fu, H. 1999. Suppression of apoptosis signal-regulating kinase 1 -induced cell death by 14-3-3 proteins. Proc. Natl. Acad. Sci. U. S. A. 96:8511-8515.

14. Zhang, R., et al. 2003. AIP1 mediates TNF- $\alpha-$ induced ASK1 activation by facilitating dissociation of ASK1 from its inhibitor 14-3-3 J. Clin. Invest. 111:1933-1943. doi:10.1172/ JCI200317790.

15. Liu, Y., Yin, G., Surapisitchat, J., Berk, B.C., and Min, W. 2001. Laminar flow inhibits TNFinduced ASK1 activation by preventing dissociation of ASK1 from its inhibitor 14-3-3. J. Clin. Invest. 107:917-923.

16. Jones, B.E., and Czaja, M.J. 1998. Mechanisms of hepatic toxicity. III. Intracellular signaling in response to toxic liver injury. Am. J. Physiol. Gastrointest. Liver Physiol. 275:G874-G878.

17. Lei, K., and Davis, R.J. 2003. JNK phosphorylation of Bim-related members of the Bcl-2 family induces Bax-dependent apoptosis. Proc. Natl. Acad. Sci. U. S. A. 100:2432-2437.

18. Zha, J., Harada, H., Yang, E., Jockel, J., and Korsmeyer, S.J. 1996. Serine phosphorylation of death agonist BAD in response to survival factor results in binding to 14-3-3 not Bcl- $\mathrm{X}_{\mathrm{L}}$. Cell. 87:619-628.

19. Datta, S.R., et al. 2000. 14-3-3 proteins and survival kinases cooperate to inactivate $\mathrm{BAD}$ by $\mathrm{BH} 3$ domain phosphorylation. Mol. Cell. 6:41-51.

\title{
Arginase: marker, effector, or candidate gene for asthma?
}

\section{Donata Vercelli}

Arizona Respiratory Center, College of Medicine, University of Arizona, Tucson, Arizona, USA

\begin{abstract}
Microarray analysis of the expression profiles of lung tissue in two murine models of asthma revealed high levels of arginase I and arginase II activity, in association with IL-4 and IL-13 overexpression (see the related article beginning on page 1863), suggesting that arginine pathways are critical in the pathogenesis of asthma.
\end{abstract}

J. Clin. Invest. 111:1815-1817 (2003). doi:10.1172/JCI200318908.

Despite intense research efforts, asthma remains a major medical and scientific challenge. Prevalence of this disease increased 75\% between 1980 and 1998 . Although this rate may now be stabilizing, the 2001 National Health Interview Survey estimated that $6.9 \%$ of adults, and $8.9 \%$ of children under the age of 18 in the United States, suffered from

Address correspondence to: Donata Vercelli, College of Medicine, University of Arizona, 1501 North Campbell Avenue, Suite 2349, Tucson, Arizona 85724-5030, USA. Phone: (520) 626-6387; Fax: (520) 626-6970; E-mail: donata@resp-sci.arizona.edu.

Conflict of interest: The author has declared that no conflict of interest exists.

Nonstandard abbreviations used: protein inhibitor of activated STAT1 (PIAS1). asthma (1). The reasons why asthma prevalence has been on the rise for so long remains a matter of intense speculation. The pathogenetic mechanisms of the disease, and the contributing genetic factors, also remain elusive. This state of affairs probably reflects the inherent complexity of the disease, and the difficulty associated with stringently defining asthmatic phenotypes so that homogenous subject groups can be identified for mechanistic studies.

\section{Microarrays: a powerful tool to dissect asthma}

An aggressive approach to the identification of new asthma genes is discussed in this issue of the JCI by Zimmermann and collaborators (2), who determined transcript expression profiles in lung tissue from mice with an asthma-like phenotype induced by sensitization with OVA or Aspergillus fumigatus. The recognized strength of microarray experiments lies in their ability to address an issue globally, and highlight the unexpected. The results of this study are no exception. An important quantitative finding was that $6.5 \%$ of the 12,422 genes analyzed showed a greater than twofold change in expression in challenged mice. These data show that, although asthma remains confined to the lung, the mechanistic dysregulation underlying the disease - whatever that may be - mobilizes a vast genetic program. Even more importantly, among the 496 and 527 genes identified in the OVA and Aspergillus models, respectively, only 291 were common to both. Since all mice had the same genetic background, this pattern is likely to result from differences in pathogenetic mechanisms, possibly related to the nature of the allergen and/or the immunization route. Such data should provide molecular epidemiologists and clinicians interested in asthma with spicy food for thought.

\section{Enter arginine and its pathways}

Intriguing findings also came from the qualitative analysis of lung transcript 


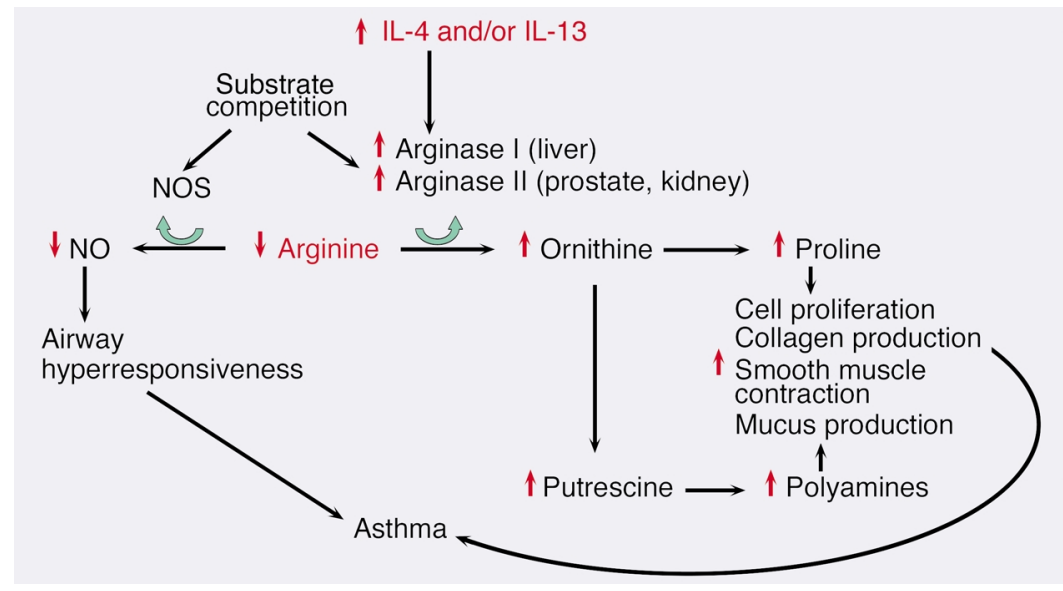

Figure 1

Arginine, arginase, and asthma. Arginase I and arginase II control the transformation of arginine into ornithine, which in turn gives rise to proline and polyamines. These products have multiple effects on connective tissue, smooth muscle, and mucus synthesis. Arginine also serves as a substrate for $\mathrm{NO}$ synthase (NOS), which generates NO, a critical regulator of airway physiology. The NOS and arginase pathways interfere with each other through substrate competition. Th2 cytokines induce arginase expression. During allergic inflammation, increased IL-4 and/or IL-13 expression results in increased expression of arginase and amplification of the arginase-dependent pathway, with concomitant suppression of $\mathrm{NO}$ generation. This leads to airway hyperresponsiveness and increased generation of mucus and collagen, all of which may contribute to the pathogenesis of asthma. The red arrows mark the upregulatory or downregulatory events that occur in arginine metabolism following increased expression of Th2 cytokines.

profiles. The genes differentially expressed in challenged mice included arginase I, arginase II, and the L-arginine transporter cationic amino acid transporter-2. All of these molecules are involved in arginine metabolism (Figure 1). In particular, arginase catalyzes the hydrolysis of arginine to ornithine and urea, and exists in two isoforms. Arginase I participates in the urea cycle, and is expressed at high levels in the liver. Arginase I deficiency results in argininemia, a disorder characterized by mental impairment, growth retardation, spasticity, and sometimes fatal episodes of hyperammonemia. Arginase II is most highly expressed in the prostate and kidney, and poorly expressed in the liver. Arginase II is thought to be involved in the synthesis of proline and/or polyamines (e.g., putrescine, spermidine, and spermine), which control cell proliferation and collagen production. To date, no human disease has been associated with a deficiency in arginase II (3).

Arginine, a molecule of many trades Of note, arginine serves as a substrate for both arginase and NO synthase (Figure 1). The arginase and NO synthase pathways can therefore interfere with one another through substrate competition (3). NO is a ubiquitous gaseous molecule that regulates many aspects of human airway biology, including airway and vascular smooth muscle tone (4). An increased concentration of $\mathrm{NO}$ in exhaled air is now recognized as a critical component of the asthmatic phenotype (5). The links with the NO pathway and collagen generation make arginine metabolism a rising star in the asthma firmament. Arginase I and arginase II were recently shown to contribute to the development of mouse lung fibrosis (6). Increased arginase activity underlies allergen-induced deficiency of $\mathrm{NO}$ and airway hyperresponsiveness in a guinea pig model of allergic asthma (7). Perhaps even more importantly, arginase expression appears to be controlled by Th2 cytokines, central mediators of allergic asthma (8). IL-13-mediated induction of arginase I in macrophages has been implicated in IL-13-dependent inhibition of NO production (9), which in turn may contribute to asthma pathogenesis. We may infer that NO inhibition resulted from substrate competition, because expression of arginase, but not NO synthase, was altered in the lungs of the allergen-challenged mice (2). Last, but not least, at the molecular level, arginine appears to be a key regulator of signaling through the JAK/STAT pathway. Indeed, posttranslational modification (methylation) of a highly conserved arginine residue in the $\mathrm{N}$-terminal domain of STAT1 is a requirement for IFN- $\alpha-$ and IFN- $\beta$-induced transcription (10). In the absence of arginine methylation, STAT1-DNA binding is impaired due to an increased association of the protein inhibitor of activated STAT1 (PIAS1) (11) with phosphorylated STAT1 dimers. No STAT6-specific PIAS has been identified to date, but the negative regulation of STAT signaling is expected to involve more than one member of the PIAS family. The search is on, and may well be successful.

\section{A novel asthma gene?}

The study by Zimmermann et al. (2) confirms that expression of arginase is increased in the asthmatic lung through a Th2-induced, STAT6-dependent mechanism, and most importantly extends these findings to humans. Interestingly, in situ hybridization in the lung of asthmatic patients revealed expression of arginase not only in submucosal inflammatory cells (most likely macrophages, as observed in the murine model) but also in airway epithelium, suggesting an even broader pattern of dysregulation. In the scenario proposed by Zimmermann and collaborators, a Th2 cytokine-dependent increase of arginase expression in the lung would affect arginine metabolism, and contribute to asthma pathogenesis through inhibition of NO generation and alterations of cell growth and collagen deposition. Thus, arginase would act as an effector of Th2 activation. The available arginase I and arginase II knock-out mice $(12,13)$, and conditional and/or tissue-specific knockouts generated ad hoc, may serve to highlight the effector role of arginase. The real question, however, is whether arginase will make it onto the list of asthma genes. The attribution of a 
causative role to arginase will depend on the results of the genetic studies that Zimmermann and colleagues' work warrants. Do single nucleotide polymorphisms in human arginase dysregulate expression and/or function so as to contribute to asthma pathogenesis? The role of arginase in the realm of asthma will ultimately be dictated by the answer to this question.

1. 2003. Asthma Prevalence, Health Care Use and Mortality, 2000-2001. National Center for Health Statistics, Center for Disease Control.

2.Zimmermann, N., et al. 2003. Dissection of experimental asthma with DNA microarray analysis identifies arginase in asthma pathogen- esis. J. Clin. Invest. 111:1863-1874 doi:10.1172/JCI200317912.

3. Morris, S.M., Jr. 2002. Regulation of enzymes of the urea cycle and arginine metabolism. Annu. Rev. Nutr. 22:87-105.

4. Fischer, A., Folkerts, G., Geppetti, P., and Groneberg, D.A. 2002. Mediators of asthma: nitric oxide. Pulm. Pharmacol. Ther. 15:73-81.

5. Wechsler, M.E., et al. 2000. Exhaled nitric oxide in patients with asthma: association with NOS1 genotype. Am. J. Respir. Crit. Care Med. 162:2043-2047.

6. Endo, M., et al. 2003. Induction of arginase I and II in bleomycin induced fibrosis of mouse lung. Am. J. Physiol. Lung Cell. Mol. Physiol. doi:10.1152/ ajplung.00434.2002.

7. Meurs, H., et al. 2002. Increased arginase activity underlies allergen-induced deficiency of cNOSderived nitric oxide and airway hyperresponsiveness. Br. J. Pharmacol. 136:391-398.

8. Wei, L.H., Jacobs, A.T., Morris, S.M.J., and Ignarro, L.J. 2000. IL-4 and IL-13 upregulate arginase I expression by cAMP and JAK/STAT6 pathways in vascular smooth muscle cells. Am. J. Physiol. Cell. Physiol. 279:C248-C256.

9. Chang, C., Zoghi, B., Liao, J.C., and Kuo, L. 2000. The involvement of tyrosine kinases, cyclic $\mathrm{AMP} /$ protein kinase A, and $\mathrm{p} 38$ mitogen-activated protein kinase in IL-13-mediated arginase I induction in macrophages: its implications in IL-13-inhibited nitric oxide production. J. Immunol. 165:2134-2141.

10. Mowen, K.A., et al. 2001. Arginine methylation of STAT1 modulates IFNalpha/beta-induced transcription. Cell. 104:731-741.

11. Liu, B., Gross, M., ten Hoeve, J., and Shuai, K 2001. A transcriptional corepressor of Stat 1 with an essential LXXLL signature motif. Proc. Natl. Acad. Sci. U. S. A. 98:3203-3207.

12. Shi, O., Morris, S.M.J., Zoghbi, H., Porter, C.W., and O'Brien, W.E. 2001. Generation of a mouse model for arginase II deficiency by targeted disruption of the arginase II gene. Mol. Cell. Biol. 21:811-813.

13. Iyer, R.K., et al. 2002. Mouse model for human arginase deficiency. Mol. Cell Biol. 22:4491-4498.

\title{
An eye on insulin
}

\author{
Sarah K. Bronson, ${ }^{1}$ Chad E.N. Reiter, ${ }^{1}$ \\ and Thomas W. Gardner ${ }^{1,2}$
}

${ }^{1}$ Department of Cellular and Molecular Physiology, and

${ }^{2}$ Department of Ophthalmology, Penn State College of Medicine, Hershey,

Pennsylvania, USA

Diabetic retinopathy, the most frequent complication of diabetes and leading cause of vision loss, involves vascular and neural damage in the retina. Insulin and IGF-1 signaling are now shown (see the related article beginning on page 1835) to contribute to retinal neovascularization, in part, by modulating the expression of various vascular mediators.

J. Clin. Invest. 111:1817-1819 (2003). doi:10.1172/JCI200318927.

Physiologic and pathologic blood vessel growth patterns are stimulated by local and systemic influences. The hope of angiogenesis research is to understand these complex interactions in order to provide better means to control pathologic vessel forma-

\footnotetext{
Address correspondence to: Thomas W. Gardner, Department of Cellular and Molecular Physiology, and Department of Ophthalmology, Penn State College of Medicine, 500 University Drive, Hershey, Pennsylvania 17033, USA.

Phone: (717) 531-6711;

Fax: (717) 531-7667;

E-mail: tgardner@psu.edu.

Conflict of interest: The authors have declared that no conflict of interest exists. Nonstandard abbreviations used: retinopathy of prematurity (ROP); diabetic retinopathy (DR); hypoxia-inducible factor-1 (HIF-1); endothelin-1 (ET-1); insulin receptor (IR); insulin-like growth factor-1 receptor (IGF-1R), vascular endothelial insulin receptor knockout (VENIRKO); vascular endothelial insulin-like growth factor receptor knockout (VENIFARKO).
}

tion, or perhaps stimulate appropriate vessel growth, to reduce maladaptive consequences. In the retina, normal vessel growth occurs in the plane of the retina from the optic nerve toward the periphery in a radial pattern and is guided by cues from astrocytes in the inner retina (1). This growth is mediated by VEGF and other ligands (2), while angioblasts from the circulation can provide endothelial progenitors (3).

\section{The problem of retinal neovascularization}

Pathologic neovascularization of the retina is a common and serious complication of retinopathy of prematurity (ROP) and diabetic retinopathy (DR). The treatment of DR, ablation of the diseased retina with laser photocoagulation or cryotherapy to cause involution of the new vessels, has remained fundamentally unchanged for almost 50 years. The nature of the growth promoting stimuli is not well understood, but in ROP the stimulus is assumed to be in part due to perinatal retinal hyperoxia followed by hypoxia. The classic response to hypoxia includes hypoxiainducing factor-1 (HIF-1) translocation to the nucleus and subsequent downstream events such as the upregulation of VEGF, eNOS, and endothelin-1 (ET-1). The fact that VEGF is increased in the vitreous of diabetic patients makes it tempting to speculate that diabetes induces a hypoxic, or HIF-1-driven response that is similar to that observed in ROP. Current animal models of diabetes do not develop proliferative retinopathy and the only model that simulates the neovascularization seen clinically is that induced by relative hypoxia in developing retinas. Postnatal mice are placed in hyperoxic conditions for several days, at a time when their vessels have not yet reached the peripheral retina, which causes vasoconstriction; when they are returned to room air, the vasoconstriction is relieved and neovascularization develops when the retina perceives relative hypoxia (4). This model provides a rodent model of neovascularization in the absence of systemic metabolic defects due to insulin depletion or resistance. Smith et al. (5) previously showed that an IGF-1 inhibitor blocked neovascularization in this model but no studies have examined the effect of the insulin receptor. 